\title{
Engagement décennal ou endettement étudiant? Les bi-admis ENS Cachan/HEC face au choix de leur école et de leur carrière
}

Getting into debt or getting paid. How elite students in business administration choose their academic and carrier paths

Morgane Le Breton et Hugo Harari-Kermadec

\section{OpenEdition}

Journals

Édition électronique

URL : http://journals.openedition.org/rfp/4230

DOI : $10.4000 /$ rfp.4230

ISSN : 2105-2913

Éditeur

ENS Éditions

Édition imprimée

Date de publication : 15 décembre 2013

Pagination : 69-80

ISBN : 978-2-84788-521-7

ISSN : 0556-7807

Référence électronique

Morgane Le Breton et Hugo Harari-Kermadec, « Engagement décennal ou endettement étudiant ? Les bi-admis ENS Cachan/HEC face au choix de leur école et de leur carrière ", Revue française de pédagogie [En ligne], 184 | 2013, mis en ligne le 15 décembre 2016, consulté le 19 avril 2019. URL http://journals.openedition.org/rfp/4230; DOI : 10.4000/rfp.4230 


\section{Engagement décennal ou endettement étudiant? Les bi-admis ENS Cachan/HEC face au choix de leur école et de leur carrière}

\section{Morgane Le Breton et Hugo Harari-Kermadec}

Dans un contexte de mise en concurrence internationale des grands établissements universitaires, l'instauration ou le relèvement des frais de scolarité semble être une évolution inéluctable. Les Écoles normales supérieures (ENS), avec leurs élèves fonctionnaires, apparaissent singulières. Dans cet article, nous étudions comment le coût des études intervient dans les choix des étudiants. Une série d'entretiens semi-directifs menés auprès d'étudiants admis à la fois à l'ENS Cachan et à l'École des hautes études commerciales de Paris (HEC) à l'issue de classes préparatoires commerciales nous permet d'appuyer et d'illustrer nos analyses sur une population pour laquelle l'accès au crédit est assuré. Nous nous intéressons en particulier à ce qui se joue, au-delà de l'aspect purement économique, dans les représentations que se font les étudiants de leurs études. Nous montrons que la question du coût interfère fortement avec celle des débouchés, une interaction anticipée par les étudiants au moment de leur choix de parcours dans l'enseignement supérieur. Dès lors, la perspective d'un emploi fortement rémunérateur imposé par l'endettement étudiant peut-elle apparaître pour certains comme une contrainte bien plus lourde qu'un engagement décennal signé avec l'État?

Mots-clés (TESE) : droits de scolarité, école payante, établissement d'enseignement supérieur, facteur économique, choix d'une école, marché de l'éducation.

\section{INTRODUCTION}

Depuis la massification de l'enseignement et la diversification des scolarités qu'elle entraîne, la sociologie s'attelle à " penser les choix scolaires " (Blanchard \& Cayouette-Remblière, 2011). Les inégalités face aux principales étapes de l'orientation (en fin de $3 e$ par exemple ; Chauvel, 2011) peuvent être interrogées sous ce prisme. La situation des classes populaires (Palheta, 2011 ; Poullaouec, 2011) comme celle des classes moyennes supérieures (van Zanten,
2010) sont régulièrement analysées. Les politiques publiques favorisent désormais ces choix, par exemple celui de l'établissement par l'assouplissement de la carte scolaire (Ben Ayed, 2011), dans un discours de responsabilisation des élèves et des familles. Ces politiques, qui font progressivement advenir La nouvelle école capitaliste (Laval, Clément, Dreux et al., 2012), prennent largement appui sur une littérature économique qui présente l'éducation comme un investissement, tant pour les États que pour les familles. 
Les choix scolaires répondent-ils à une logique d'investissement ? La question mérite d'être posée, du moins pour ce qui concerne l'enseignement supérieur. Tandis que la hausse des frais de scolarité est récente dans certains pays de I'OCDE tels que le Canada (Frenette, 2007 ; Ouellet \& Martin, 2011) ou l'Angleterre (Callender \& Jackson, 2008 ; Casta, 2010), dans d'autres, l'université est payante depuis longtemps (Chili, États-Unis). Aux États-Unis, après une hausse continue depuis plusieurs décennies, le coût annuel de la scolarité dans les collèges publics (établissements locaux d'un enseignement supérieur court, que l'on pourrait rapprocher des Instituts universitaires de technologie [IUT]) est passé, entre 1975 et 2005 , de 10 à 21 semaines de salaire moyen, et de 21 à 53 semaines dans les collèges privés (Vinokur, 2009 , p. 445). Dans ces conditions, le calcul économique occupe nécessairement une place prépondérante dans les processus de choix des étudiants.

En France, l'université est légalement gratuite, ou plutôt soumise à des tarifs réglementés et faibles puisqu'ils sont limités à quelques centaines d'euros. Mais depuis quelques années, de nombreux arguments sont mis en avant pour justifier la hausse du tarif légal appliqué aux universités, voire pour appeler à la dérégulation des frais. Au-delà de l'argument souvent avancé de l'insertion sur un marché international de l'enseignement supérieur aux prix bien plus élevés, le manque de moyens (Gary-Bobo \& Trannoy, 2005) et le caractère anti-redistributif d'un enseignement supérieur quasi gratuit majoritairement suivi par les enfants des classes supérieures (Courtioux, 2009, 2010 ; Gregoir, 2008) sont les plus régulièrement invoqués. Les effets réels qu'aurait une telle évolution en France restent en discussion (voir Flacher, HarariKermadec \& Moulin, 2012, pour une revue de la littérature).

Cependant, certains établissements d'enseignement supérieur échappent déjà au régime réglementé, parfois depuis longtemps, à commencer par les écoles de commerce qui pratiquent des frais significatifs depuis plusieurs décennies. En effet, depuis quelques années, on assiste à une augmentation importante des frais dans les plus prestigieuses de ces écoles, à leur généralisation à toute la filière gestion', mais également au-delà, comme à l'Institut d'études politiques (IEP) de Paris (SciencesPo) dont les tarifs annuels hors décote pour 2014-2015 sont de $9940 €$ pour le collège universitaire (cycle licence) et de $13700 €$ pour le master². C'est encore en gestion que sont apparus les premiers masters payants dans une université publique, l'université
Paris-Dauphine (jusqu'à $4000 €$ depuis la rentrée 2010-2011). Les écoles d'ingénieurs emboîtent le pas aux écoles de commerce : le tarif légal, fixé à $584 €$ par an en 2012, est respecté par un peu plus de la moitié des 149 écoles d'ingénieurs recensées par l'Étudiant (écoles en 3 et en 5 ans), 34 pratiquent des frais compris entre ce tarif légal et $2500 €$ (surtout des écoles en 5 ans) et 35 autres se situent entre $4000 €$ et $8500 €$ annuels.

À l'opposé de ces importantes hausses des frais de scolarité, les Écoles normales supérieures (ENS) offrent, en plus d'un très haut prestige intellectuel, un statut de fonctionnaire stagiaire rémunéré $(1300 €$ nets mensuels) à leurs élèves, en échange d'un engagement décennal à travailler pour l'État français ${ }^{3}$ ou dans l'enseignement supérieur et la recherche à l'international. Dans cet article, nous nous intéressons à des étudiants ayant à choisir entre ces deux extrêmes puisqu'ils sont à la fois admis à l'École des hautes études commerciales de Paris (HEC) et à l'École normale supérieure de Cachan (ENSC). En effet, le recrutement en sortie de classes préparatoires étant une procédure concurrentielle, les meilleurs élèves de deuxième année de classes préparatoires économiques et commerciales ("prépa HEC ») peuvent être admis dans la plus prestigieuse des écoles de commerce et dans une ENS. Cette situation est même courante puisque le concours d'entrée à l'ENSC de cette filière s'appuie sur les résultats des écrits d'HEC4.

Les bi-admis ENSC/HEC doivent donc faire un choix que nous analysons selon un point de vue d'économiste : endettement étudiant ou engagement décennal ? Pour répondre à cette question, nous confronterons les principales hypothèses de la théorie économique standard à une série d'entretiens avec des bi-admis. Par la suite, nous étudierons plus particulièrement le(s) rôle(s) que peuvent jouer les frais de scolarité dans la prise de décision. Enfin, nous montrerons que l'augmentation de ces frais, en plus de modifier mécaniquement les paramètres du choix, transforme qualitativement les déterminants de la décision.

\section{CAPITAL HUMAIN OU CAPITAL CULTUREL ?}

La théorie économique néoclassique propose un cadre général d'analyse de l'éducation à partir du concept de capital humain (Becker, 1964). Dans ce cadre, l'éducation est une transformation de la future 
force de travail qui permet d'en améliorer la productivité. C'est en ce sens qu'il s'agit d'un capital, qu'il faut investir dans l'humain à l'aide d'une dépense d'éducation pour en espérer des retours, qui peuvent être à la fois privés (pour le diplômé) et sociaux (pour la collectivité). En plus de peser dans l'appréhension académique des questions d'éducation, ce cadre théorique est au cœur des politiques éducatives internationales (notamment au niveau de l'OCDE) et européennes (Laval, Clément, Dreux et al., 2012). Nous nous proposons donc de discuter de la pertinence de cette théorie pour décrire et analyser les déterminants des choix des étudiants rencontrés au cours d'une enquête par entretiens. La population cible de notre enquête, admise à HEC, est en effet l'une des rares pour lesquelles il est légitime de lever la principale objection opposée à la théorie du capital humain : la contrainte d'accès au crédit pour les étudiants. Cette contrainte et sa levée ont suscité une abondante littérature (Carneiro \& Heckman, 2002 ; Fernandez, 1998 ; Gregoir, 2008 ; Keane \& Wolpin, 2001) sur différentes formules de crédit étudiant et diverses expériences nationales. En France, le crédit étudiant est en pratique très limité du fait de l'existence d'une filière universitaire aux faibles droits d'inscription et d'un accès des milieux populaires à l'enseignement supérieur relativement faible. Notre terrain est donc un observatoire privilégié pour confronter la théorie économique standard aux comportements et aux représentations des étudiants face à l'opportunité de financer, éventuellement à crédit, des études coûteuses mais rentables.

\section{MÉTHODOLOGIE ET PÉRIMÈTRE DE L'ÉTUDE}

Pour cette étude, une série d'entretiens semi-directifs ont été conduits auprès de sept personnes concernées par ce choix entre HEC et l'ENSC. Le recours à des entretiens semi-directifs permet de comprendre en profondeur les mécanismes sousjacents à la sélection d'une école, sans a priori et en laissant libre la réponse de l'interlocuteur.

Lors de la construction de notre échantillon, nous avons cherché à disposer de toute la variabilité disponible dans une population source assez limitée : le dernier intégré à l'ENSC est usuellement classé entre les 20 e et 30 e rangs et n'est pas nécessairement admis à HEC. Les bi-admis sont donc tout au plus une vingtaine chaque année. Ils partagent tous un cursus scolaire de très haut niveau et sortent des meilleures classes préparatoires économiques et commerciales. Pour les élèves d'HEC, l'accès à la liste des candidats originaires de ces classes, admis mais non intégrés à l'ENS de Cachan, a permis de constituer la première partie de l'échantillon. Quant aux élèves de l'ENS, nous avons fait appel en premier lieu à des contacts personnels avant d'élargir le champ à des promotions d'élèves différentes ; enfin un dernier étudiant s'est présenté spontanément. Toutes les propositions d'entretiens adressées ont été acceptées, ce qui témoigne d'une forte disponibilité de la part de ces élèves à revenir sur leur choix d'orientation.

L'échantillon ainsi constitué est mixte, tant du point de vue des études que du sexe, puisqu'il est composé de deux normaliennes et deux normaliens (admis aux concours 2007, 2010 et 20115) ainsi que d'une étudiante et deux étudiants à HEC (admis aux concours 2008, 2009 et 2011), chacun ayant été simultanément admis aux deux écoles lors du concours 6 .

Tous sont issus de classe préparatoire aux grandes écoles (CPGE) commerciale, quatre pour la voie scientifique et trois pour la voie économique (deux normaliens et une étudiante d'HEC). Quelques traits caractéristiques de ces individus peuvent être dressés afin de les définir plus nettement et de soulever les différences notoires qui existent entre eux ou visà-vis du reste de la population étudiante. Premièrement, la population enquêtée ici constitue une élite académique ayant suivi une classe préparatoire et intégré une très grande école, si bien qu'une sélection s'est déjà opérée en faveur des classes sociales les plus privilégiées (Duru-Bellat \& Kieffer, 2008). Ensuite, deux témoins ont " cubé "7 et un troisième s'est réorienté vers la voie commerciale après une année en classe préparatoire scientifique. Ces trois étudiants sont élèves d'HEC. Un normalien a fait un an de classe préparatoire littéraire avant de se réorienter tandis que trois autres ont intégré l'ENSC après le parcours standard de deux années de CPGE commerciale. En outre, un élève seulement (HEC) a totalement accompli sa scolarité à Paris, mais il est à noter que deux individus sont enfants de militaires et ont voyagé durant toute leur enfance. Ils ne peuvent pas être rattachés à une ville française de référence, même s'ils vivaient à Paris les années précédant leur CPGE. À l'inverse, trois étudiants se revendiquent ouvertement " de la province » et sont tous les trois normaliens. Le dernier étudiant considéré a réalisé l'ensemble de sa scolarité dans une grande ville de province. 
Comme attendu, tous disposent d'un fort capital scolaire et culturel. Plusieurs étudiants ont suivi une scolarité en section européenne anglais et tous ont obtenu la mention Très bien au baccalauréat sans avoir redoublé au cours de leur scolarité 8 , un étudiant ayant sauté une classe (normalien). Tous les étudiants interrogés ont présenté au minimum une option pour le baccalauréat (principalement le latin), à laquelle certains ont ajouté une option culturelle supplémentaire (danse, musique, etc.). Enfin, au sein de cette population, un individu est boursier (normalien). Quatre témoins ont deux parents en emploi et trois un père actif et une mère inactive (deux sans emploi, une retraitée).

À partir de cet échantillon réduit, nous nous proposons d'analyser plus spécifiquement trois entretiens, dans le but de cerner quelques trajectoires particulières et néanmoins caractéristiques, au vu de notre enquête. Cette restriction vise à permettre d'expliciter au mieux le raisonnement et les motivations de chacun, en les confrontant aux hypothèses de la théorie du capital humain. Les quatre entretiens restants sont mobilisés plus ponctuellement, pour compléter l'analyse. Les trois étudiants retenus sont Anne $^{9}$, élève en $2^{2}$ année à l'ENS Cachan au moment de l'enquête (printemps 2012) et issue du concours 2010, Paul, élève de 1re année à l'ENS Cachan lors du témoignage et Sophie, entrée à HEC en 2011.

Anne est originaire de la région parisienne mais a voyagé au cours de son enfance du fait de la profession de son père (ingénieur militaire). Sa mère est, comme son père, titulaire d'un master et se trouve actuellement sans emploi après un poste de cadre commercial d'entreprise privée. Ses parents ont été très attentifs à sa réussite scolaire, ce dont témoigne le choix des établissements (en privilégiant ceux réputés d'un bon niveau). Le contexte familial d'Anne ne la destinait pas en premier lieu à choisir une carrière dans le public et, en outre, intégrer HEC aurait certainement été mieux valorisé dans son entourage. En revanche, la connaissance de la carrière de ses parents a développé chez elle une certaine aversion au risque, avec l'idée de pouvoir conserver un emploi en toutes circonstances.

Paul a grandi dans une grande ville de province jusqu'à son intégration à l'ENS Cachan. Ses parents appartiennent au milieu enseignant : son père est agrégé et sa mère enseignante-chercheuse. II hérite, de ses parents, d'une vision favorable à l'égard de la fonction publique. Ces valeurs l'emportent au moment du choix sur des considérations plus économiques puisque, selon son propos, le niveau de vie qu'il peut espérer dans la fonction publique n'est pas comparable à celui qui prévaudrait avec une carrière dans le privé. À l'instar d'Anne, le fait de ne pas avoir vécu dans la capitale l'éloigne quelque peu des schémas habituels de réussite scolaire et sociale qui placeraient HEC comme le summum de la réussite française dans le domaine économique et commercial.

Les deux derniers normaliens interrogés ont grandi en province dans deux villes de taille moyenne. Leur origine sociale ne les destinait pas à une telle réussite scolaire. Ils ne font pas état de l'intervention de leurs parents - une mère cadre dans le public et un père dirigeant de PME pour l'un ; des parents travaillant dans le domaine social pour l'autre - dans leur choix d'école, ce qui est ici étroitement associé à une très faible information familiale sur les grandes écoles. C'est donc à partir de leur goût pour les études que ces deux normaliens se sont orientés. Les déterminants évoqués lors des entretiens portent sur le contenu des enseignements et ne relèvent pas d'une logique économique. Ils présentent dès lors un intérêt moindre dans la perspective développée dans cet article. Nous en retenons principalement que des étudiants peuvent être indifférents aux débouchés professionnels à l'heure du choix d'école, tout du moins dans la situation où ils sont assurés a minima d'un accès aisé à un statut d'enseignant.

Sophie, qui a choisi HEC, a également voyagé en raison de la carrière de son père (colonel) et sa mère est sans emploi. Ses parents ont dû se contenter d'études courtes du fait du manque de moyens financiers de leurs propres parents, c'est pourquoi ils ont à l'inverse souhaité que Sophie puisse faire ses choix sans se préoccuper du coût de ses études. Elle avait tout d'abord opté pour une classe préparatoire littéraire mais, n'étant pas attirée par ce qu'elle nomme "le monde de la connaissance pure ", a assez vite développé une volonté de travailler en droit des affaires (domaine sélectif et fortement rémunérateur), ce qui l'a conduit à rejoindre une filière économique et commerciale afin d'intégrer HEC.

Les deux autres élèves d'HEC interrogés sont des enfants d'anciens fonctionnaires passés dans le privé. Le père du premier est devenu entrepreneur à la suite d'une carrière militaire (sa mère est retraitée après une carrière militaire également). Les parents du second ont quitté des postes de chercheurs pour devenir cadres de grandes entreprises. Même si ce dernier hérite d'une vision favorable envers le milieu de la recherche, qu'il n'exclut pas rejoindre un jour, ils 
partagent fondamentalement avec Sophie la même analyse quant à l'orientation. En effet, ils se caractérisent par une forte attention portée aux classements d'écoles, la volonté d'appartenir à une élite sociale, de " faire carrière " et d'obtenir des rémunérations élevées, des aspirations conformes aux positions sociales de leurs parents.

\section{LE CHOIX D'ÉCOLE EST-IL RATIONNEL ?}

Dans sa théorie du capital humain, G. Becker se place dans un cadre néoclassique de marché pour l'éducation et la formation et souhaite expliquer ce qui motive la demande sur ce marché. II considère en effet que l'éducation et la formation directe dans l'entreprise, mais aussi la santé, constituent des moyens d'un processus général, l'investissement en capital humain : "for some purposes schools can be treated as a special kind of firm and students as a special kind of trainee " (Becker, 1964, p. 52). II définit ce capital de la façon suivante: " activities that influence future monetary and psychic income by increasing the resources in people. These activities are called investments in human capital " (Becker, 1964, p. 11). Pour l'auteur, chacun opte pour un investissement optimal en capital humain sur la base d'un arbitrage coûts/ bénéfices. Un individu choisira de s'éduquer si cet investissement s'avère plus rentable qu'un placement financier de même montant et qu'une participation plus précoce au marché du travail, c'est-à-dire si la somme actualisée des flux de revenus futurs, nette des coûts d'opportunité, est supérieure en investissant dans son capital humain que sur un produit financier. II affirme en effet que : " the most important single determinant of the amount invested in human capital may well be its profitability or rate of return " (Becker, 1964, p. 59). Plus précisément : « an informed, rational person would invest only if the expected rate of return was greater than the sum of the interest rate on riskless assets and the liquidity and risk premiums associated with the investment " (Becker, 1964, p. 91).

Économiquement, les étudiants bi-admis ont le choix entre, d'une part, payer des frais de scolarité élevés pendant trois ans à HEC pour ensuite mener une carrière fortement rémunératrice de cadre dirigeant ou d'entrepreneur et, d'autre part, recevoir un salaire de fonctionnaire stagiaire pendant quatre ans à la condition de travailler par la suite six années supplémentaires pour l'État en échange d'un revenu relativement limité. L'existence même d'étudiants choisissant I'ENSC met en difficulté le modèle, tandis que le cas des étudiants d'HEC est a priori plus favorable à ce cadre théorique. Néanmoins, nous montrerons que les étudiants intégrant HEC ont également pris en compte d'autres aspects que la somme actualisée de leurs revenus futurs pour s'orienter.

L'accès à une information complète pose évidemment problème dans le processus de formulation du choix, même dans le cas des très grandes écoles qui nous intéresse ici : plusieurs étudiants reviennent dans les entretiens sur les difficultés rencontrées et mentionnent l'absence de recours dans leur entourage. L'effet du capital social, à travers la familiarité ou au contraire la distance entre l'étudiant et l'école, sur la capacité à s'approprier l'information mériterait une discussion que nous préférons laisser de côté pour nous concentrer sur l'hypothèse de rationalité, elle-même fortement contestable. Comme l'énonce M. Gurgand : " L'évaluation des différents déterminants des niveaux d'éducation repose ici sur un cadre d'analyse qui suppose les agents hyperrationnels, capables d'évaluer les coûts et les avantages intertemporels de chaque choix à chaque instant " (Gurgand, 2005).

Cette présentation de l'hypothèse de rationalité souligne son caractère caricatural et trivialement irréaliste. Surtout, elle suppose que le raisonnement se réduit au calcul du gain économique net tiré des études. Des raisonnements de ce type apparaissent dans certains entretiens lorsqu'il est question du choix de l'école, mais rarement comme emportant la décision. Ainsi, Iorsqu'on demande à Anne si elle a choisi l'ENSC pour le salaire de normalienne, elle répond:

« Ha non! C'est une chose que j'ai voulu éliminer. Je ne voulais pas choisir l'ENS uniquement parce que j'étais payée, ça me semblait une très très mauvaise incitation parce qu'au final, à HEC, j'aurais fini par travailler dans le privé, c'est sûr, parce qu'il aurait fallu rembourser $40000 €$, donc j'aurais forcément gagné plus, enfin je pense. En termes futurs, j'aurais plus gagné en étant allée à HEC que là, en étant à l'ENS. Clairement le salaire ça n'aurait pas du tout été la même chose. Mais bon, je le sais. Et ça ne me dérange pas tellement. "

Anne a donc conscience qu'elle ne choisit pas la filière la plus rémunératrice mais la préfère pour d'autres raisons (souhait de mener une vie moins stressante, intérêt pour les métiers de la sphère publique). On retrouve sensiblement le même raisonnement pour Paul qui avance sa proximité avec les 
valeurs associées à la fonction publique en opposition à celles qui prévalent dans le secteur du commerce ; il adhère en outre aux conséquences qu'il considère liées au statut de fonctionnaire (accepter d'être moins rémunéré que dans le privé au profit d'un cadre de vie plus serein et/ou avec l'opportunité de développer son savoir - en cas de carrière d'enseignant-chercheur - par exemple). Enfin, comme présenté en introduction, les deux autres normaliens indiquent préférer à l'enrichissement monétaire un enrichissement intellectuel qu'ils ne pensent pas trouver dans la formation professionnelle dispensée par HEC. Les débouchés de cette école leur semblent par ailleurs plus stressants que ceux de l'ENSC. Le choix de l'ENSC, que la théorie du capital humain échoue à expliquer, repose sur des déterminants sociaux. Paul ayant des parents fonctionnaires, il aspire à retrouver pour lui-même un mode de vie et un ensemble de valeurs compatibles avec ce choix de carrière. À l'inverse, Anne cherche plutôt à se détacher du modèle de ses parents pour se prémunir des aléas d'une carrière dans le privé dont ils ont eu à souffrir ; elle est attirée par l'emploi à vie garanti par la fonction publique. Une autre normalienne imite également le choix de ses parents en intégrant la fonction publique, ce qu'elle justifie par son souhait de se préserver d'une carrière stressante. Le dernier normalien paraît tourné vers la volonté d'enrichir sa connaissance et ne mentionne pas de préoccupation pour une imitation ou au contraire un détachement du cadre familial. Cela peut se justifier par le fait que sa mère est fonctionnaire tandis que son père ne l'est pas, et qu'ils n'ont vraisemblablement pas transmis un modèle unique à leur fils.

Anne, Paul et les autres normaliens indiquent donc explicitement qu'il ne s'agit pas d'un choix économique. Les termes employés par cette étudiante en économie - « incitation », " en termes futurs »révèlent une appropriation de la théorie du capital humain et certainement d'une formalisation a posteriori des choix d'école (choix réalisés avant la découverte de la théorie). On peut supposer que c'est cette familiarité avec la théorie économique qui la conduit à s'en dissocier aussi explicitement. On peut également noter qu'elle ne formule pas dans notre entretien d'explication à ces choix à partir des outils disciplinaires dont elle dispose. II ne s'agit pas d'un manque de réflexivité dans la mesure où elle repousse spontanément ces outils dans son discours ; il s'agit plutôt d'un choix relevant d'un registre externe à l'objet des sciences économiques qu'elle a apprises.

Le calcul économique "rationnel » semble donc incapable de rendre compte du choix de l'ENSC. Un élément du choix, omis et pourtant décisif, apparaît dans d'autres entretiens : l'« envie d'apprendre » l'emporte - à en croire un normalien - sur le supplément de revenu, dans une situation où un niveau de vie conforme aux aspirations est de toute façon garanti. C'est donc à l'accumulation de capital culturel en elle-même que cet étudiant s'attelle, plutôt qu'à une accumulation instrumentalisée de connaissances en vue de leur rentabilisation postérieure, ou a fortiori à un détournement de l'institution scolaire à des fins de signalement (Spence, 1973) que nous analyserons plus loin.

À l'inverse, les individus ayant opté pour HEC tiennent un discours beaucoup plus conforme à l'hypothèse de rationalité. Sophie, ayant intégré HEC en 2011, a dû faire face au choix d'école quelques mois seulement avant notre entretien. Si, comme Anne, elle fait usage spontanément d'éléments de théorie économique lorsqu'elle est interrogée au sujet de la rémunération à l'ENS, c'est au contraire en les reprenant à son compte. Pour elle, le raisonnement semble conforme à la logique développée par la théorie du capital humain, et un constat très pragmatique ressort de sa formalisation du choix. Ce dernier se rapproche véritablement d'une analyse " rationnelle " dans laquelle elle a réussi à se projeter et qui lui permet de formuler les différents enjeux découlant de son choix :

" Je regardais les salaires à la sortie d'HEC, qui sont assez élevés! Surtout dans la filière droit, plus qu'en finance ! C'est un investissement pour l'avenir en somme, on étale le revenu. "

Pour Sophie, des éléments de calcul économique sont donc de nature à fonder son choix d'école. Toutefois, tous trois rapportent de surcroît que la notoriété de l'école a beaucoup compté dans leur réflexion, mettant en évidence que leur hésitation aurait été plus grande s'ils n'avaient pas eu HEC mais une école moins bien classée. En outre, on peut citer Sophie qui décrit son arbitrage entre HEC, les autres écoles de commerce et l'ENSC, fortement influencé par un classement de ces écoles :

"Si je n'avais pas eu HEC ou l'ESSEC, j'aurais pris l'ENS je pense. La plus-value d'HEC a fait la différence au niveau réputation mais pas toutes les écoles de commerce. C'est vrai que quand on dit l'ENS... "

Cet arbitrage exprimé en termes de notoriété est sans doute facilité par un environnement familial privilégié qui s'attache à donner de l'importance à la renommée des établissements fréquentés, plus qu'au contenu réel de la formation. Ce critère se justifie d'autant plus que, vivant à Paris depuis quelques 
années avant d'effectuer son choix d'école, Sophie a pu être plus influencée par le poids accordé à la notoriété de l'établissement.

Les entretiens des deux autres étudiants ayant choisi HEC font apparaître les mêmes déterminants que Sophie dans le choix de l'école : ils se réclament également d'un calcul économique et de la notoriété d'HEC. Comme elle, ils vivent en centre-ville, à Paris pour l'un, dans une grande ville de province pour l'autre. Tous trois reconnaissent que la perspective de carrière à l'issue d'HEC et la rémunération associée justifient l'investissement pour les études et valent les dépenses engagées.

II est donc fait ici référence au paiement des frais de scolarité comme à un investissement qui portera ses fruits plus tard, dans une optique parfaitement beckerienne. Celle-là semble donc apte à rendre compte d'une partie seulement des étudiants, ceux dont la préférence va pour HEC. Les frais de scolarité interviennent pourtant dans le choix de l'ENSC, mais à travers des raisonnements d'une toute autre nature.

\section{DÉSISTEMENT À HEC : LA CONTRAINTE N'EST PAS CELLE QUE L'ON CROIT}

Pour certains étudiants, le calcul économique est décisif et s'accompagne d'un argumentaire qui peut être très précis. C'est par exemple ce que rapporte Anne, dans un calcul très complet :

« II fallait que je m'endette pour aller à HEC parce que mes parents n'auraient jamais payé et ça m'embêtait beaucoup parce que tu t'endettes sur 30000 euros pour ta scolarité et puis il faut payer ton logement, qui se monte quand même à 550 euros par mois, donc si tu fais le calcul, ça va très vite : tu rajoutes 15000 euros. Donc tu empruntes 45000 euros... Et je ne voyais pas comment rembourser. Donc il aurait fallu travailler, mais travailler depuis Jouys-en-Josas, sans le permis, tu ne peux pas, or je n'avais pas le permis et je n'avais pas les moyens de le payer, donc il aurait fallu que j'emprunte encore plus ! Je ne m'en serai jamais sortie dans le remboursement, ou alors si, ça aurait pris 10 ans comme les autres. En fait, je ne connais personne qui soit allé à HEC et qui se soit vraiment endetté. »

Cet extrait fait apparaître avec force la longue réflexion d'Anne quant à l'endettement et à son influence sur son choix d'école. Une réflexion marquante puisqu'elle est capable d'en rendre compte de façon très détaillée et ponctuée de chiffres précis énoncés sur un ton assuré et rapide. Paul est tout à fait dans la même situation. Pour l'un comme pour l'autre, l'indépendance financière vis-à-vis de la famille - permise par le statut de normalien - est de plus déterminante. Bien qu'il soit peu vraisemblable que leurs familles aient fait face à d'importantes difficultés économiques dans la mesure où les deux parents travaillent et occupent des professions intermédiaires ou supérieures, les valeurs de responsabilité et d'autonomie économiques ont occupé une place importante. Ainsi, au cours de leur scolarité préalable, Paul et Anne ont travaillé durant leurs vacances d'été pour disposer d'un budget personnel durant l'année scolaire.

Les deux autres normaliens ont également travaillé durant leurs étés, mais ne mentionnent pas la question de l'endettement comme un des déterminants de leur choix d'école. Ce n'est vraisemblablement pas par désinvolture ou par facilité d'accès au crédit que l'endettement ne s'est pas posé comme un problème concret, mais plutôt par manque d'information sur HEC. L'option d'intégrer l'école de commerce a été écartée avant même que ne soit abordée la question financière sous une modalité pratique. Leurs milieux familiaux respectifs éloignés des grandes écoles (ces deux normaliens ont grandi dans des villes moyennes de province, les parents de l'un travaillent dans le secteur social et ceux de l'autre sont respectivement dirigeant d'une PME et cadre intermédiaire dans le public) les ont amenés à fonder leur choix sur des critères académiques plutôt qu'économiques. C'est donc leur intérêt pour les disciplines étudiées à I'ENSC qui est mis en avant dans les entretiens, alors que les perspectives professionnelles à la sortie des études ne sont pas mobilisées.

A contrario, les étudiants qui ont opté pour HEC n'ont pas réellement considéré la variable financière comme un paramètre dans leur arbitrage. Sophie, par exemple, fait état de ses réflexions à l'égard du critère financier. Elle a conscience de l'obstacle que ce coût peut représenter pour nombre d'étudiants mais admet qu'elle a été exemptée de cette contrainte grâce à un soutien financier familial. À notre question à propos de l'impact de la rémunération de l'ENSC sur son choix d'école, elle répond :

" [Le salaire versé par l'ENS est-il entré en ligne de compte ?] Pas trop, pas trop car je n'avais pas de difficultés financières et puis il y avait le crédit étudiant. Mon cousin était en école de commerce aussi, il a fait un prêt étudiant et était tranquille. En fait, je n'avais pas de problème de financement à la base, avec la carrière de mon père [colonel], on était tranquilles. 
C'est sûr que c'est un privilège, le critère compte pour beaucoup, mais moi j'ai eu la chance de pouvoir choisir sans la barrière financière. "

On peut noter l'importance de l'aide prodiguée par ses parents lorsque son discours à la première personne du singulier se transforme en un discours à deux voix (celle de ses parents - son père surtout - et la sienne). Elle passe effectivement de "je n'avais pas de problème de financement à la base " à " on était tranquilles » dans la même phrase. Contrairement à la situation de Paul et d'Anne, le fait de rester à la charge de ses parents - une charge renforcée par le paiement des frais d'inscription à HEC - n'est pas une préoccupation pour Sophie. On retrouve le même raisonnement chez les deux autres étudiants ayant choisi HEC, à savoir une certaine indifférence à l'égard du coût des études justifiée par un fort soutien parental.

À l'extrême opposé des discours des élèves normaliens, le critère financier est ainsi exclu du choix d'école par les étudiants ayant choisi HEC. L'un d'entre eux, pourtant candidat à I'ENSC, ignorait jusqu'à l'existence d'une rémunération des élèves normaliens. II apparaît alors que ces étudiants n'ont pas non plus choisi HEC sur la base d'un calcul économique. C'est bien au contraire une situation financière permettant de ne pas tenir compte du coût de la scolarité qui les amène à faire ce choix, sur la base d'autres critères qui restent à établir. Les références à la rentabilité sur le long terme de l'investissement éducatif apparaissent alors comme une justification a posteriori d'un choix qui ne s'est pas construit sur cette base.

Il semble donc qu'au sein de notre échantillon d'étudiants relativement favorisés et à l'avenir professionnel assuré, une importante différence de capital économique intervienne pour distinguer les étudiants ayant choisi HEC de ceux ayant préféré I'ENSC, en complément d'appréciations distinctes vis-à-vis de la fonction publique et du savoir universitaire. Le coût de la scolarité, qui englobe non seulement les frais d'inscription mais également les frais d'entretien de la vie quotidienne (logement, restauration, transport, sorties qui revêtent une place prépondérante dans la socialisation à $\mathrm{HEC}$, etc.), se révèle discriminant socialement malgré un accès au crédit facilité permettant, d'un point de vue strictement économique, de faire face à ces dépenses. II s'agit dès lors de s'interroger sur les causes de cette aversion pour l'endettement chez les futurs normaliens, qui souscrivent dans le même temps à une autre contrainte : l'engagement décennal.
Contrairement à ce que laissaient supposer les extraits précédents ainsi que le modèle de Becker, le long terme et l'anticipation de l'entrée sur le marché du travail peuvent intervenir en faveur du choix de I'ENSC. L'endettement nécessaire à l'intégration d'HEC est en effet rejeté à cause de ses répercussions à long terme sur le choix de carrière : afin de rembourser l'emprunt, il aurait fallu renoncer à une carrière dans le public sous peine de rallonger la durée et le coût du remboursement. C'est en substance le propos tenu par Paul qui met en lumière les implications pesant sur son choix d'école. La contrainte de moyen terme sur son choix de carrière rattrape ainsi sa capacité de financement éventuelle à court terme (même s'il explique les efforts qu'il aurait dû faire pour payer les frais de scolarité d'HEC) :

" J'avais une petite réserve d'argent qui faisait que je pouvais le faire... Si je travaillais l'été et que mes parents finançaient les dépenses quotidiennes, je pouvais payer un peu moins de la moitié des frais de scolarité, mais je n'avais pas envie de débuter ma vie active endetté jusqu'au cou. [...] Cela signifie qu'au moment où tu fais ton choix de carrière, tu es sous contrainte. Certes, l'emprunt est indolore mais il va falloir que je choisisse tel type de poste. Si je veux bosser dans l'administration, t'es moins bien payé. Je rembourse comment? Je n'avais pas trop envie de me retrouver pris au piège. "

Paul fait apparaître la réelle contrainte financière qui incombe aux étudiants aspirant à une carrière publique : le renoncement à cette carrière du fait de l'insuffisance de la rémunération obtenue par un fonctionnaire pour rembourser le prêt contracté pour intégrer HEC. En fait, l'aspiration à travailler dans le public supplante la maximisation des revenus qu'il aurait pu toucher en optant pour HEC, et c'est en ce sens que l'engagement décennal n'est plus vu comme une contrainte car les élèves normaliens souhaitent in fine travailler pour l'État. La contrainte financière paraît donc anticipée par les étudiants interrogés concernés (c'est-à-dire les non-boursiers ne disposant pas d'un soutien familial permettant d'éviter de recourir à un emprunt pour s'acquitter des frais de scolarité) au moment d'intégrer une école et pèse fortement sur leur choix. Peu avant, Paul reliait les enjeux éthiques qui ont influencé son choix d'école et les enjeux financiers sous-jacents. Pour lui, les deux aspects sont intimement liés et il dira plus tard que c'est en réalité cette imbrication des enjeux qui l'a fait pencher pour l'ENSC:

"Et puis il y avait un enjeu financier aussi, mais qui est lié. C'est-à-dire que quand tu rejettes ce monde-là, un des aspects qui fait que tu le rejettes est aussi un 
Tableau 1. Évolution des frais d'inscription dans 6 écoles de commerce entre 2008 et 2011

\begin{tabular}{|l|c|c|c|c|}
\hline & Rentrée 2008 & Rentrée 2011 & Évolution & Rentabilité \\
\hline ESC Toulouse & $8500 €$ & $9900 €$ & $16 \%$ & 3,3 \\
\hline EM Lyon & $7900 €$ & $11200 €$ & $42 \%$ & 3,2 \\
\hline EDHEC & $8900 €$ & $11200 €$ & $26 \%$ & 3,2 \\
\hline ESCP Europe & $8400 €$ & $11500 €$ & $37 \%$ & 3,2 \\
\hline HEC & $10300 €$ & $11900 €$ & $16 \%$ & 3,0 \\
\hline ESSEC & $10500 €$ & $15000 €$ & $43 \%$ & 2,4 \\
\hline
\end{tabular}

Source : GOURDON Jessica (2011), letudiant.fr. En ligne : <http://www.letudiant.fr/etudes/ecole-de-commerce/ecoles-de-commerce-les-fraisde-scolarite-senvolent-et-lessec-franchit-un-seuil-14214.html>.

Note : la colonne «Rentabilité » donne le rapport entre le salaire moyen en sortie d'école et les frais de scolarité de la dernière année d'étude.

aspect pratique qui te désincite. On peut choisir l'administration après HEC, mais être obligé de donner des cours de maths à Intégrale pour rembourser l'emprunt, c'est quand même un peu un échec... »

Ce constat est relatif au même type de raisonnement et éclaire différemment le problème, car il met en évidence le refus de participer à un système fondé sur les appareils lucratifs comme les cours particuliers qui seraient une des seules solutions pour un fonctionnaire pour pouvoir rembourser l'emprunt contracté. Le travail purement lucratif, rejeté lors le choix de la fonction publique à la sortie d'HEC, se serait de toute façon imposé à Paul sous la forme de l'impératif d'un remboursement à effectuer.

Finalement, la contrainte financière est double : à court terme et également à plus long terme, en filigrane des choix de carrière. Les boursiers sont quant à eux exemptés de frais de scolarité à HEC, mais il n'en demeure pas moins qu'ils doivent s'acquitter des dépenses annexes précédemment citées. Leur montant étant loin d'être négligeable, elles font partie de l'arbitrage entre la scolarité à HEC et à l'ENS.

\section{QUAND LES FRAIS AUGMENTENT... HEC N'EN DEVIENT QUE PLUS ATTRACTIVE}

Déjà importants, les frais de scolarité des écoles de commerce sont en augmentation rapide. Nous reproduisons ici un extrait d'un tableau de l'Étudiant indiquant les évolutions des frais des grandes écoles de commerce (voir tableau 1) ${ }^{10}$.
Le tableau 1 présente une forte augmentation et une certaine convergence des frais dans ces écoles autour de $11500 €$ correspondant à une rentabilité légèrement supérieure à 3 , c'est-à-dire à un salaire annuel en sortie d'école de l'ordre de $35000 €$. Ce phénomène correspond au mécanisme d'imitation entre écoles positionnées sur un même " marché " décrit par Blanchard (2012) : plutôt que de s'adapter à une demande difficile à cerner (et, on le verra, hétéronome dans ses choix), les "entreprises " se copient les unes les autres pour s'assurer de rester dans la course. Le calcul et l'exposition d'une rentabilité par le site letudiant.fr nous semblent révélateurs de la diffusion de la logique beckerienne : c'est en tant qu'investisseurs que les étudiants sont interpellés par le journal. Pourtant, nous l'avons dit, ce raisonnement ne semble pas déterminant dans les choix des étudiants que nous avons interrogés. Au contraire, plutôt que de s'inquiéter d'une augmentation des frais qui viendraient réduire la rentabilité de leur investissement, les étudiants d'HEC s'y avèrent favorables.

Nous distinguons deux éléments pouvant jouer dans ce sens. D'une part, un coût élevé de la formation peut apparaître comme un marqueur de qualité pour les étudiants : en supposant implicitement un mécanisme de marché, le " prix » élevé de la formation révèle qu'elle est préférée aux autres par les " consommateurs". Le prix des études joue alors le rôle de la renommée de l'établissement ou du diplôme.

Ce mécanisme peut agir de façon indirecte, les élèves cherchant à anticiper les préférences de leurs futurs recruteurs en choisissant la formation qu'ils jugent la plus appréciée par eux, et qui devient donc également celle qui est préférée par les étudiants. 
Sophie évoque ce raisonnement en mentionnant le classement qu'elle a elle-même constitué au moyen d'éléments subjectifs et objectifs. Sa hiérarchisation est nette :

« Si je n'avais pas HEC et l'ESSEC, j'aurais choisi I'ENS. Mais là, la plus-value d'HEC a fait la différence au niveau de la réputation. Quand on dit l'ENS, c'est certes connu mais moins qu'HEC. J'ai bien vu que les recruteurs se basent sur un nom de diplôme, et qu'ils n'en ont rien à faire de ce que tu fais concrètement. "

Le classement de Sophie est donc constitué à partir de l'idée qu'elle se fait de celui des recruteurs, un classement peu sensible au contenu des enseignements. On retrouve en tout point un raisonnement similaire de la part des autres étudiants en commerce, qui portent une attention particulière à l'image renvoyée par leur(s) diplôme(s), l'un d'entre eux allant même parler d'une "inflation au diplôme de qualité " à propos des doubles cursus suivis dans le but de se différencier des étudiants ayant étudié dans la même institution principale. Cette prégnance du signal peut s'expliquer par le fait que les trois étudiants d'HEC interrogés proviennent d'un milieu à fort capital social et misant sur les titres scolaires dans une perspective de reproduction sociale. À la différence des normaliens, le choix est donc dicté par la volonté d'être reconnu par les employeurs et ne semble pas reposer sur l'intérêt porté au contenu des études.

D'autre part, la possibilité offerte par HEC de réaliser une importante dépense d'éducation peut être lue dans l'optique de la théorie du signal (Spence, 1973). Dans ce cadre théorique, les études ne modifient pas la productivité des étudiants mais leur permettent de signaler leur " talent intrinsèque »-qu'ils sont supposés connaître. Une formation gratuite n'a alors aucun attrait puisqu'elle ne signale aucune disposition à payer pour révéler son talent. Le diplôme et les études ne garantissent plus un certain niveau de productivité mais sont davantage un signal de différenciation pour les employeurs, afin de les aider à contrer la sélection adverse (Akerlof, 1970). Ce n'est alors plus tant la qualité de l'école qui justifie un coût élevé, mais bien le prix du bien HEC qui en constitue la qualité, appuyée sur une position de leader en termes de classement des écoles de commerce.

Par ailleurs, la dépense que constitue le paiement des frais de scolarité peut être considérée non plus comme un investissement éducatif mais comme une dépense de consommation. HEC apparaît alors comme un bien de luxe, et suivre cette formation devient alors une forme de consommation ostentatoire (Veblen, 1899). Ainsi, " consommer " le bien
HEC permet de se signaler auprès de ses pairs et de se distinguer du reste de la population, afin de se positionner dans une catégorie sociale dont le capital économique est élevé. Cela expliquerait l'indifférence de certains étudiants quant à une croissance des frais de scolarité, qui renforce au contraire le caractère ostentatoire de la consommation. La préférence pour des frais encore plus élevés (élasticité de la consommation par rapport au prix positive) est justement le marqueur du caractère luxueux du bien.

Si l'on ne peut réduire les déterminants du choix d'HEC à cet effet de démonstration (Duesenberry, 1949, pour la version macroéconomique de la théorie de Veblen) du statut social, nous trouvons dans nos entretiens une version inversée de ce mécanisme. Ainsi, si notre enquête n'a pas fait apparaître de motivations ostentatoires (peut-être difficiles à assumer en entretien) parmi les principales explications du choix d'HEC, certains étudiants mentionnent un rejet du statut social identifié à cette école justifiant de s'en dissocier, ce refus conduisant de façon symétrique à un effet de contre-sélection. En témoigne Paul, qui revient sur cet aspect « ostentatoire » du choix :

" HEC c'est plus reconnu que D2 [section économiegestion de l'ENS Cachan], mais cela permet justement de dire "Je suis dans une école, je fais de l'économie et de la gestion", donc de garder la tête froide. C'est très bien comme ça. Je crois que j'aurais été mal à l'aise d'être catalogué en disant tout le temps "Je suis à HEC". "

II affirme ainsi un élément annexe qui a tout de même pesé dans son choix : le fait que son école reflète en partie qui il est. II refusait l'image qui l'aurait caractérisé s'il avait opté pour HEC à laquelle il ne s'identifiait pas. Si Anne partage à nouveau ce raisonnement tenu par Paul, les deux autres normaliens ne le développent pas dans la mesure où ils portent peu d'intérêt à l'image renvoyée par chacune des deux écoles et ont arrêté leur choix à partir du contenu des enseignements.

\section{CONCLUSION : EFFET RETOUR}

L'évolution rapide de la tarification des écoles de commerce accroît la sélection sociale à l'entrée, en renforçant des mécanismes d'orientation scolaire au sein même des étudiants au capital scolaire le plus élevé. La théorie du capital humain n'apparaît pas à même de rendre compte de ces mécanismes, qui 
relèvent pourtant du champ économique par la présence de frais d'inscription et d'entretien élevés. Les critiques usuelles de cette théorie - la présence d'une contrainte d'endettement, le manque de ressources cognitives des agents - ne semblent pas déterminantes dans notre configuration privilégiée. Des stratégies de reproduction (ou d'évitement) sociale semblent prépondérantes dans le choix de l'ENSC, perçue comme ouvrant sur des carrières publiques quand HEC paraît conduire à des postes plus rémunérateurs mais plus instables. L'indépendance financière assurée par le statut de normalien est un élément important de ce choix ; l'importance accordée à cette autonomie au cours d'études élitistes ne s'explique pas aisément par l'origine sociale de ces étudiants, dont les parents occupent des professions intellectuelles supérieures et disposent de moyens économiques conséquents. Une enquête plus approfondie pourrait remonter à la génération précédente pour analyser la transmission des valeurs relatives à l'indépendance économique.

Les étudiants choisissant HEC, à l'inverse, ne semblent pas se préoccuper du soutien économique de leurs parents. Le choix scolaire est finalement dicté par le futur recruteur : les étudiants choisissent HEC parce qu'ils anticipent que cette école sera mieux valorisée lors de l'arrivée sur le marché du travail. Si ce comportement correspond davantage à une vision de l'étudiant comme agent rationnel maximisateur de revenu inter-temporel, nous n'avons pas observé de situations de réel arbitrage : les coûts liés à la scolarité sont externalisés vers les parents, et l'éventuelle rémunération de normalien ne présente qu'un intérêt limité, quand elle n'est pas ignorée.

Notre enquête fait apparaître une distinction forte entre les étudiants choisissant HEC et l'ENSC, se structurant autour d'un rapport opposé vis-à-vis de l'indépendance financière, articulé à des aspirations sociales divergentes. Dans les deux cas, on peut supposer que l'origine sociale marque ces représentations, bien qu'elle soit relativement homogène dans notre échantillon (parents cadres, souvent de la fonction publique pour une partie du moins de leur carrière dans les deux groupes). C'est peut-être dans l'analyse de la trajectoire sociale familiale sur plusieurs générations qu'il faut chercher l'explication de ces valeurs qui participent à la réduction de la mobilité sociale dans un contexte de massification et de libéralisation universitaire.

Morgane Le Breton
Morgane.le-breton@ens-cachan.fr
ENS Cachan
Hugo Harari-Kermadec
Hugo.harari@ens-cachan.fr
ENS Cachan, IDHES et CEPN

Hugo Harari-Kermadec ENS Cachan, IDHES et CEPN

\section{NOTES}

1 L'ensemble des écoles de commerce forment environ 20000 étudiants par promotion.

2 Faut-il y voir la confirmation de l'analyse d'A. Garrigou (2003) : "Sciences-Po rêve d'être une "business school" "?

3 Y compris les entreprises de statut privé à capital majoritairement public.

4 II s'agit de la série D2 option 2, 3, 4 (économie-gestion) qui ouvre chaque année une dizaine de postes du département économie-gestion de l'ENSC aux élèves admissibles (c'est-à-dire invités à passer les oraux) à l'une des trois grandes écoles de commerce parisiennes (HEC, ESSEC, ESCP).

5 Deux témoins ont intégré l'ENS Cachan en 2010. L'un des normaliens (concours 2007) s'est présenté spontanément pour l'étude après avoir appris l'existence de ce projet. Cette démarche montre le poids d'un tel choix d'école dans la vie des étudiants et la volonté d'apporter leur témoignage à ce sujet. " Je me suis dit que peut-être je pouvais t'apporter quelque chose, [...et j'avais] le souhait d'être utile et d'aider une personne travaillant sur un sujet qui me parle et m'intéresse (je me pose pas mal de questions à propos des choix d'école, etc.) " Pour ne pas risquer de biaiser l'étude par ce témoignage spontané, il a fallu s'assurer qu'il ne connaissait pas les conclusions du travail, qu'il n'avait pas eu accès à la grille d'interview, et les questions posées étaient parfois différentes de la grille standard pour compléter les apports précédents.

6 La profonde crise économique marquant ces années n'est mentionnée par aucun des étudiants. Nous remercions le rapporteur anonyme ayant soulevé ce point, que nous ne pouvons expliquer que par la situation privilégiée de ces bi-admis.

7 C'est-à-dire redoublé leur deuxième année de CPGE commerciale.

8 L'un d'entre eux a redoublé la grande section de maternelle pour passer outre le CP par la suite, dans un contexte très spécifique, c'est pourquoi nous n'avons pas relevé ce « redoublement ".

9 Par souci d'anonymat, les trois prénoms attribués sont fictifs.

10 La dernière colonne, qui indique le rapport entre salaire de sortie et frais annuels, sera commentée dans la dernière section. 


\section{BIBLIOGRAPHIE}

AKERLOF G. (1970). "The Market for "Lemons": Quality Uncertainty and the Market Mechanism ». The Quarterly Journal of Economics, vol. 84, no 3, p. 488-500.

BECKER G. (1964). Human capital: a theoretical and empirical analysis, with special reference to education. New York : NBER Columbia University Press.

BEN AYED C. (2011). «À qui profite le choix de l'école? Changements d'établissement et destins scolaires des élèves de milieux populaires ". Revue française de pédagogie, no 175, p. 39-57.

BLANCHARD M. (2009). "From "Écoles Supérieures de Commerce" to "Management Schools": transformations and continuity in French business schools ». European Journal of Education, vol. 44, no 4, p. 586-604.

BLANCHARD M. (2012). Socio-histoire d'une entreprise éducative : le développement des Écoles supérieures de commerce en France (fin $d u$ xIxe siècle-2010). Thèse de doctorat, sociologie, École des hautes études en sciences sociales.

BLANCHARD M. \& CAYOUETTE-REMBLIÈRE J. (2011). "Penser les choix scolaires ». Revue française de pédagogie, no 175, p. 5-14.

CALLENDER C. \& JACKSON J. (2008). " Does the fear of debt constrain choice of university and subject of study? ». Studies in Higher Education, vol. 33, no 4, p. $405-429$.

CARNEIRO P. \& HECKMAN J. (2002). "The Evidence on Credit Constraints in Post-Secondary Schooling ». The Economic Journal, vol. 112, no 482, p. 705-734.

CASTA A. (2010). "Frais d'inscription et prêts étudiants en Angleterre : discours, origines et effets ". L'Homme et la société, vol. 178, no 4, p. 51-74.

CHAUVEL S. (2011). «Auto-sélections et orientation en fin de 3 e : réflexions issues d'une enquête de terrain ". Revue française de pédagogie, no 175, p. 85-88.

COURTIOUX P. (2009). "Peut-on financer l'éducation du supérieur de manière plus équitable ? ". EDHEC position paper.

COURTIOUX P. (2010). « Une contribution des diplômés du supérieur à leurs études est-elle envisageable ? ". Revue française d'économie, vol. 24, no 3, p. 121-165.

DUESENBERRY J. (1949). Income, saving and the theory of consumer behavior. Cambridge (États-Unis) : Harvard University Press.

DURU-BELLAT M. \& KIEFFER A. (2008). « Du baccalauréat à l'enseignement supérieur en France : déplacement et recomposition des inégalités ". Population, vol. 63, no 1 , p. $123-157$.
FERNANDEZ R. (1998). Education and Borrowing Constraints: Tests vs. Prices. National Bureau of Economic Research. En ligne : < http://www.nber.org/papers/ w6588>.

FLACHER D., HARARI-KERMADEC H. \& MOULIN L. (2012) «Faut-il (vraiment) augmenter les frais d'inscription à l'université ? ". Revue française d'économie, vol. 27, no 3, p. 145-183.

FRENETTE M. (2007). «Pourquoi les jeunes provenant de familles à plus faible revenu sont-ils moins susceptibles de fréquenter l'université ? Analyse fondée sur les aptitudes aux études, l'influence des parents et les contraintes financières ". Statistique Canada. Direction des études analytiques : document de travail, no 295.

GARRIGOU A. (2003). "Sciences-Po rêve d'être une "business school" ". Manière de voir, no 72.

GARY-BOBO R. \& TRANNOY A. (2005). « Faut-il augmenter les droits d'inscription à l'université ? ". Revue française d'économie, vol. 19, no 3, p. 189-237.

GREGOIR S. (2008). «Les prêts étudiants peuvent-ils être un outil de progrès social ? ". EDHEC position paper.

GURGAND M. (2005). Économie de l'éducation. Paris : La Découverte.

KEANE M. \& WOLPIN K. (2001). « The effect of parental transfers and borrowing constraints on educational attainment ". International Economic Review, vol. 42, no 4, p. 1051-1103.

LAVAL C., CLÉMENT P., DREUX G. \& VERGNE F. (2012). La nouvelle école capitaliste. Paris : La Découverte.

OUELLET M. \& MARTIN E. (2011). Université inc. Des mythes sur la hausse des frais de scolarité et l'économie du savoir. Québec : Lux.

PALHETA U. (2011). " Enseignement professionnel et classes populaires : comment s'orientent les élèves "orientés" ". Revue française de pédagogie, no 175, p. 59-72.

POULLAOUEC T. (2011). "Choix du destin et destin du choix ". Revue française de pédagogie. no 175, p. 81-84.

SPENCE M. (1973). « Job Market Signaling ». The Quarterly Journal of Economics, vol. 87, no 3, p. 355-374.

VEBLEN T. (1899). The theory of the leisure class: An economic theory of institutions. New York : Macmillan.

van ZANTEN A. (2010). "Choix de l'école et inégalités scolaires ». Agora débats/jeunesses, vol. 56, no 3, p. 35-47.

VINOKUR A. (2009). " La querelle des droits d'inscription. Quel financement pour sauver le service public à l'Université ? ». Revue du MAUSS, no 33, p. 337-349. 